\title{
Loss of JNK-Associated Leucine Zipper Protein Promotes Peritoneal Dialysis-Related Peritoneal Fibrosis
}

\author{
Maoqing Tian ${ }^{\mathrm{a}}$ Lu Zhang $^{\mathrm{a}}$ Yujuan Wang $^{\mathrm{a}}$ Meili Deng $^{\mathrm{a}}$ Cancan Peng $^{\mathrm{a}}$ \\ Wei Liang $^{\mathrm{a}}$ Guohua Ding $^{\mathrm{a}}$ Bo Shen ${ }^{\mathrm{b}}$ Huiming Wang ${ }^{\mathrm{a}}$

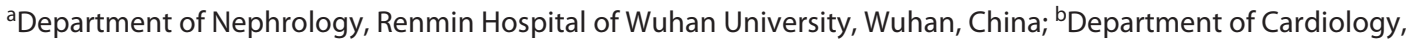 \\ Renmin Hospital of Wuhan University, Wuhan, China
}

\section{Keywords}

Peritoneum fibrosis - JNK-associated leucine zipper protein .

Transforming growth factor- $\beta 1$ - Epithelial mesenchymal transition

\begin{abstract}
Background: Peritoneal dialysis-related peritoneal fibrosis is the leading cause of peritoneal ultrafiltration failure. Multitude factors and pathological processes have been implicated in peritoneal fibrosis development and progression, whereas the intrinsic anti-fibrotic mechanism has rarely been explored. JNK-associated leucine zipper protein (JLP) has been recently found possessing powerful anti-fibrotic merits of overall antagonizing TGF- $\beta$-induced profibrotic effects. Objectives: We wondered whether JLP is expressed in the peritoneum, and if so, whether it exerts the anti-fibrotic effects similar to those in the kidney. Method: Here, we examined and confirmed JLP expression in peritoneum tissue of mice. Then, we established a peritoneal fibrosis model in $J / p$ wild-type and Jlp global deficient mice and observed the different effects of Jlp on peritoneal fibrosis progression. In vitro studies were performed on peritoneal mesothelial HMrSV5 cells with or without Jlp knockdown to investigate the underlying mechanism by which Jlp exerts anti-fibrotic effects. Results: We found that the expression of JLP decreased in a high-glucose peritoneal dialysis solution
\end{abstract}

karger@karger.com www.karger.com/kdd

Karger $\stackrel{\text { ' }}{5}$

GOPEN ACCESS
(C) 2022 The Author(s)

Published by S. Karger AG, Basel

This is an Open Access article licensed under the Creative Commons Attribution-NonCommercial-4.0 International License (CC BY-NC) (http://www.karger.com/Services/OpenAccessLicense), applicable to the online version of the article only. Usage and distribution for commercial purposes requires written permission.
(HGPDS)-induced peritoneal fibrosis mouse model and in HGPDS-treated peritoneal mesothelial cell HMrSV5. JLP deletion exacerbated HGPDS-induced peritoneal fibrosis in peritoneal fibrosis mice, and knockdown of JLP resulted in an increased profibrotic response to HGPDS stimulation in HMrSV5 cells, which was associated with epithelial-to-mesenchymal transition, elevated autophagy, and apoptosis, as well as enhanced TGF- $\beta 1 /$ Smad signaling activation. Conclusions: Our findings revealed a new anti-fibrotic factor of Jlp involved in peritoneal fibrosis induction and shed light on novel therapeutic targets in peritoneal ultrafiltration failure.

(c) 2022 The Author(s)

Published by S. Karger AG, Basel

\section{Introduction}

As an important renal replacement therapy for patients with end-stage renal disease, peritoneal dialysis (PD) is applied in $11 \%$ of dialysis patients around the world [1]. Efficient and stable PD therapy relies on the structural and functional integrity of the peritoneal membrane, which is a semipermeable membrane consisting of a monolayer of peritoneal mesothelial cells resting upon a thin basement

Maoqing Tian, Lu Zhang, and Yujuan Wang share the same contribution.
Correspondence to:

Bo Shen, 574190062@qq.com

Huiming Wang, rm000301@whu.edu.cn 
membrane. However, in the process of long-term PD, the peritoneal membrane is persistently under harmful circumstances of high-glucose peritoneal dialysis solution (HGPDS), uremia, and the possible challenge of peritonitis, whereby undergo structural damage and functional impairment, ultimately culminating in irreversible peritoneal fibrosis and ultrafiltration (UF) failure $[2,3]$.

The pathogenesis of peritoneal fibrosis is an extremely complex process that involves multiple factors and cell events, including excessive activation of the transforming growth factor- $\beta$ (TGF- $\beta$ ) signaling pathway, epithelial mesenchymal transition (EMT), apoptosis, dysregulation of autophagy, and inflammatory infiltration. TGF- $\beta 1$ is a major profibrotic factor and the main molecule that causes the degeneration of peritoneal structure and function $[4$, 5]. TGF- $\beta 1$ is recognized as the main subtype of fibrotic regulation [6] and a prototypical inducer of EMT [7]. The characteristics of EMT include improved cell migration and invasion and enhanced anti-apoptotic ability and stem cell characteristics [8]. In addition, the role of TGF- $\beta 1$ in autophagy has attracted extensive attention. Autophagy is a phenomenon of self-eating within the cell, which is a defensive mechanism of cell self-degradation and recycling of intracellular components [9]. Dysregulation of autophagy involves in pathogenesis of fibrotic diseases. However, the role of autophagy and the mechanisms underlying TGF- $\beta 1$ activation in peritoneal fibrosis remain unclear.

JNK-associated leucine zipper protein (JLP, also known as SPAG9 or JIP4) is a scaffold protein for mammalian mitogen-activated protein kinase signaling pathways that belongs to the JIP (JNK interacting protein) family [10]. JLP is widely expressed in various organs, including the brain, liver, lung, heart, spleen, testis, ovary, and kidney [11] and is upregulated in various cancers, including ovarian cancer, hepatocellular carcinoma, and astrocytoma [12-14]. Considering the role of JLP in cancers, it has been recognized as a cancer biomarker [15]. JLP is a multifunctional protein that can regulate cell growth, differentiation, proliferation, invasion, and apoptosis [14]. Recently, it has also been found coordinating the cell process of autophagy [16]. Interestingly, our previous study demonstrated that JLP is involved in the regulation of TGF- $\beta$ induced renal fibrosis $[17,18]$, suggesting JLP as a potential anti-fibrosis factor. However, the role of JLP in peritoneal fibrosis remains unclear. In this study, we investigated the role and mechanism of JLP in regulation of peritoneal fibrosis. First, we validated the expression of JLP in the peritoneum. Second, peritoneal fibrosis models induced by HGPDS were established in mice and human peritoneal mesothelial cells. Third, we found that JLP has a vital effect in anti-peritoneal fibrosis via negatively regulating the TGF- $\beta 1 /$ Smad signaling pathway to counteract TGF- $\beta 1$ initiated EMT, autophagy, and apoptosis.

\section{Methods}

Reagents and Antibodies

HGPDS (4.25\%) was purchased from Baxter China. Anti-JLP antibody (ab12331) was used for Western blotting, and anti-JLP antibody (SC-271492) was used for immunofluorescence staining. The primary antibodies for Western blotting against $\alpha$-smooth muscle actin ( $\alpha$-SMA) (ab124964), fibronectin (ab45688), collagen-I (ab34710), TGF- $\beta 1$ (ab92486), phospho-Smad2 (ab188334), phospho-Smad3 (ab52903), p62/SQSTM1 (ab56416), and LC-3B (ab51520) were from Abcam (Cambridge, MA, USA). Anti-GAPDH (sc-365062) was purchased from Santa Cruz (Santa Cruz, CA, USA). Smad2/3 (D7G7), vimentin (D21H3), E-cadherin (24E10), $\mathrm{N}$-cadherin (13A9), and Beclin 1 (3738) were purchased from Cell Signaling Technology (Danvers, MA, USA). PARP1 (A19596) was obtained from ABclonal (Wuhan, China). Rabbit IgG-HRP (ANT020), mouse IgG-HRP (ANT019), and Alexa Fluor 488/594 antibodies were obtained from Antgene (Wuhan, China).

\section{Mice and Animal Models}

Wild-type (WT) and $J l p$ global deficient $\left(J l p^{-/-}\right)$mice were generated by inbreeding WT mice. Eight- to 10 -week-old weightmatched and sex-matched littermates were used in the experiments. WT and $J l p^{-/-}$mice were maintained on 12-h light/dark cycles under specific pathogen-free conditions with unrestricted access to food and water at the Center for Animal Experiments of Wuhan University. All protocols were approved by the Animal Ethics Review Board of Wuhan University and performed in accordance with the guidelines of the National Health and Medical Research Council of China.

A mouse model of HGPDS-induced peritoneal fibrosis was established as previously described [19]. 4.25\% HGPDS or PBS was intraperitoneally injected daily into male mice for 4 weeks. The peritoneal equilibration test (PET) was conducted 28 days after PD treatment as previously described [20,21]. The dialysate creatinine concentration (D), plasma creatinine concentration $(\mathrm{P})$, peritoneal fluid glucose concentration (D0), and peritoneal fluid glucose concentration (D2) were measured.

\section{Histology and Immunohistochemistry Examination}

The peritoneal tissue was fixed with $4 \%$ paraformaldehyde and then embedded. Five-micrometer-thick sections were stained with hematoxylin and eosin (HE) or IHC staining. The mean peritoneal thickness was measured in 5 visual fields, with five measurements per field.

\section{Cell Culture and Transfection}

Human peritoneal mesothelial cells (HMrSV5) were maintained at $37^{\circ} \mathrm{C}$ in $5 \% \mathrm{CO}_{2}$ in RPMI-1640 containing $10 \%$ fetal bovine serum, supplemented with penicillin and streptomycin. Cells were stimulated with $4.25 \%$ HGPDS for $24 \mathrm{~h}$. For siRNA transfection, we performed HiPerFect transfection according to the manufacturer's protocol with Jlp siRNA and control siRNA (Qiagen). The sequence of human JLP siRNA oligonucleotides was 5'-CAGACCCGAGTGGAATCTTTA-3'. 


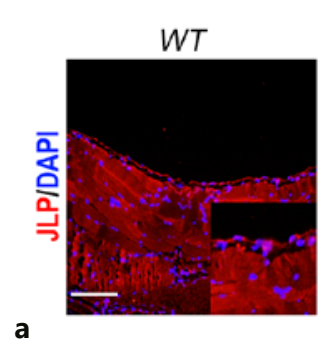

PBS,WT
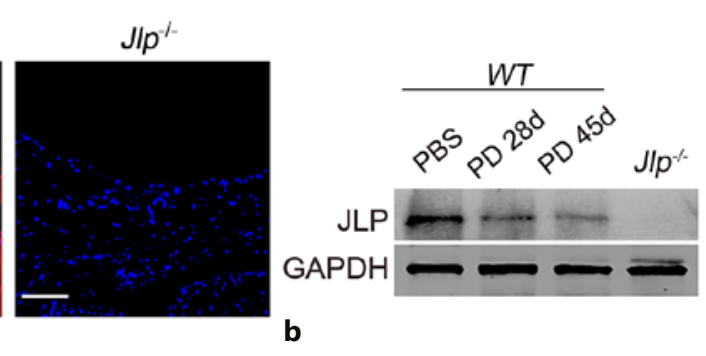

PBS, Jlp ${ }^{-/-}$

PD,WT

$\mathrm{PD}, \mathrm{Jlp} \mathrm{p}^{-/-}$
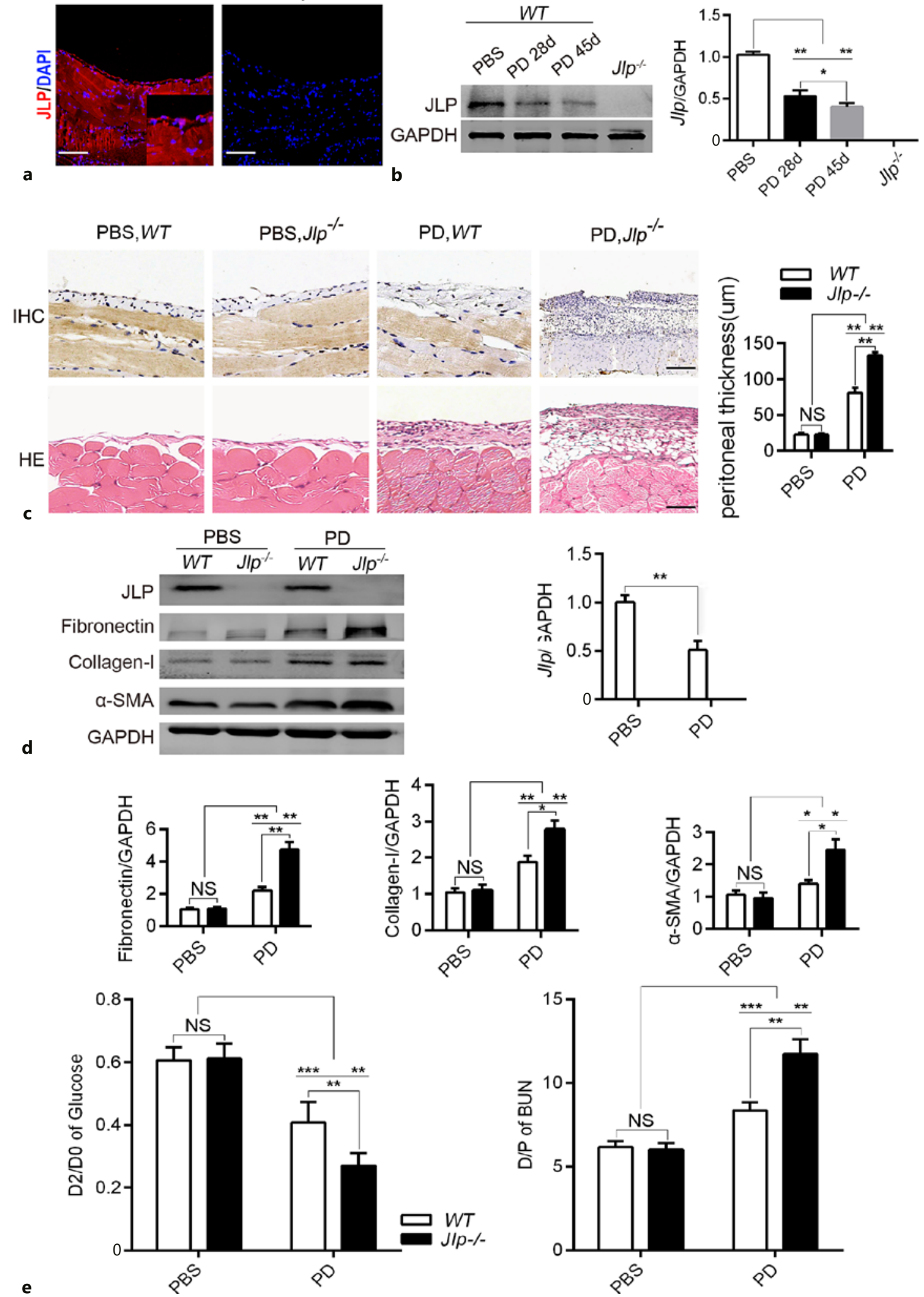

e

(For legend see next page.) 
Western Blotting

The parietal peritoneum was peeled away and lysed in lysate buffer containing protease inhibitors for the subsequent Western blotting. The peritoneal tissues and cells were lysed in lysate buffer with protease inhibitors. Proteins were separated by $8 \%$ or $12 \%$ SDS-PAGE and then transferred to a PVDF membrane (Merck Millipore). Next, the PVDF membrane was blocked with 5\% skim milk in TBST for $1 \mathrm{~h}$ at room temperature and incubated with the indicated primary antibodies and HRP-conjugated secondary antibody (IgG). Immunoreactive bands were detected with ECL reagent and scanned with Bio-Rad ChemiDoc XRS.

\section{Enzyme-Linked Immunosorbent Assay and Wound-Healing}

Assay

The secretion of TGF- $\beta 1$ was detected by using the ELISA kit (Human TGF- $\beta 1$ ELISA kit; Neobioscience, Shenzhen, China). Cell migratory abilities were tested by a wound-healing assay as described previously [22]. A wound was created by scratching with a pipette tip after transfection with Jlp siRNA or control siRNA. Cells stimulated with HGPDS or PBS were visualized by light microscopy at $0 \mathrm{~h}, 12 \mathrm{~h}, 24 \mathrm{~h}$, and $48 \mathrm{~h}$ (3 separate fields/well). Covered surface areas were measured by ImageJ.

\section{Immunofluorescence Staining}

IF staining was performed as described previously [23]. HMrSV 5 cells were fixed with 4\% paraformaldehyde for 20 min at room temperature. After washing, the cells were blocked with $5 \%$ albumin bovine $\mathrm{V}$ for $1 \mathrm{~h}$ and then incubated with the indicated primary antibodies and fluorescently labeled secondary antibodies. Cell nuclei were visualized by co-staining with DAPI. Representative photographs ( 5 visual fields for each tissue analyzed, five measurements per field) were captured using a laser scanning confocal microscope (Olympus, Tokyo, Japan).

\section{Apoptosis Assay}

Cell apoptosis assays were performed using a PE Annexin V Apoptosis Detection Kit (BD Biosciences, Franklin Lakes, NJ, USA) according to the manufacturer's instructions. In brief, cells were treated with HGPDS or PBS after $24 \mathrm{~h}$ of transfection with Jlp

Fig. 1. Jlp deficiency aggravated peritoneal fibrosis in the peritoneal fibrosis mice model. a IF staining shows the expression of JLP in a single layer of peritoneal mesothelial cells of $W T$ mice but not $J l^{-/-}$ mice. $\mathbf{b}$ Detection of the JLP protein in mouse peritoneal membrane tissue with or without PD treatment for indicated days by Western blotting, $J l p^{-/-}$group as negative control. c The parietal peritoneal membrane was obtained from $W T$ mice and $J l p^{-/-}$mice in the PD and PBS groups. Representative photographs (5 visual fields for each tissue analyzed, five measurements per field) of HE staining show that the peritoneal thickness was dramatically increased in the $J l p^{-/-}$ mice with peritoneal fibrosis compared with that in the WT mice. IHC staining showed that the levels of JLP protein were downregulated after PD treatment. Scale bar, $100 \mu \mathrm{m}, n=5$. d Western blotting analyses of the expression of the indicated proteins in the indicated peritoneal membrane. GAPDH was set as loading control. $n=3$. e PET shows the function of peritoneal permeability in WT mice and $J l p^{-/-}$mice in the PD and PBS groups. NS, no significant difference. ${ }^{*} p<0.05,{ }^{* *} p<0.01,{ }^{* * *} p<0.001$.

Loss of JLP Promotes Peritoneal Fibrosis
siRNA or control siRNA. Then, the cells were harvested and resuspended in $500 \mu \mathrm{L}$ of $1 \times$ binding buffer and then transferred to a 5 -mL culture tube containing $5 \mu \mathrm{L}$ of PE Annexin $\mathrm{V}$ and $5 \mu \mathrm{L}$ of 7 -AAD. The cells were gently vortexed and incubated for $15 \mathrm{~min}$ at room temperature in the dark. After incubation, the cells were analyzed by Accuri flow cytometry (BD Biosciences). Flow-Jo software was used for analysis of the results.

\section{Statistical Analysis}

The results are expressed as the mean \pm standard error of the mean and were analyzed using GraphPad Prism 6. One-way or twoway analysis of variance was applied to analyze data for comparisons between groups. All the data were confirmed in at least three separate experiments. $p$ values $<0.05$ were considered statistically significant.

\section{Results}

\section{Jlp Deficiency Aggravated Peritoneal Fibrosis in the}

Peritoneal Fibrosis Mice Model

We found for the first time that JLP was present in the peritoneum, predominantly expressed by peritoneal mesothelial cells (Fig. 1a). Additionally, the expression of JLP was significantly decreased in a time-dependent manner (28 and 45 days) after PD treatment (Fig. 1b). We further established an HGPDS-induced PD mouse model in WT mice and $J l p^{-/-}$mice. The expression of JLP decreased in peritonea of the peritoneal fibrosis mouse model by IHC staining, which is consistent with the Western blotting assay (Fig. 1c). Next, we examined the histologic features of peritoneal injury by HE staining. Compared with PBStreated WT mice, PD treatment exhibited increased peritoneal thickening, whereas knockout of Jlp further increased peritoneal thickening (Fig. 1d). Moreover, we found that the expression of fibronectin, collagen-I, and a-SMA was upregulated in the peritoneal membrane of the PD mouse model compared with PBS treatment mice and further increased when Jlp was knocked out (Fig. 1d). To evaluate whether Jlp deficiency worsened the peritoneal UF rate, the glucose $\mathrm{D} / \mathrm{D} 0$ ratio and $\mathrm{BUN} \mathrm{D} / \mathrm{P}$ ratio were calculated to assess water transport and small solutes in the peritoneal membrane (Fig. 1e). We found that these pathological changes aggravated by Jlp deletion were associated with increased peritoneal permeability.

\section{Jlp Knockdown Aggravated HGPDS-Induced Fibrotic}

\section{Responses in HMrSV5 Cells}

To further investigate the role of JLP in regulation of peritoneal fibrosis in vitro, $\mathrm{HMrSV} 5$ cells were treated with HGPDS by transfection with control siRNA or Jlp siRNA (Fig. 2a). The protein levels of fibronectin, collagen-I, and a-SMA were higher in HGPDS treatment compared with 


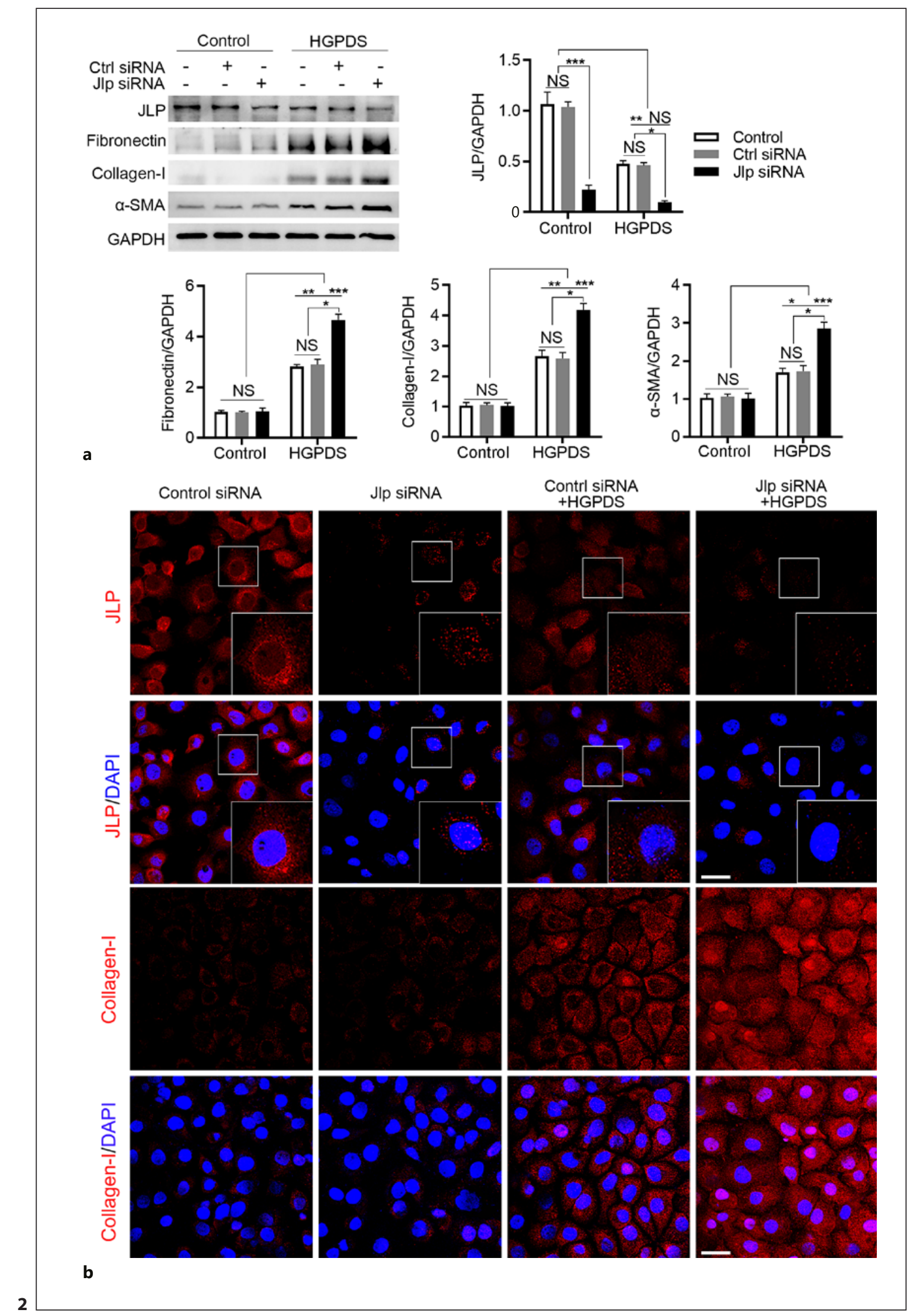

(For legend see next page.) 
the control group by Western blotting (Fig. 2a). IF staining showed the upregulation of collagen-I, which was further increased in HMrSV5 cells transfected with Jlp siRNA (Fig. 2b). These results indicated that downregulation of Jlp promotes HGPDS-induced peritoneal fibrosis.

\section{JLP Regulated Peritoneal Fibrosis via the TGF- $\beta 1 /$ \\ Smad Signaling Pathway}

It has been reported that the TGF- $\beta 1 /$ Smad signaling pathway plays a critical role in peritoneal fibrosis [4]. To investigate the potential role of JLP in regulation of peritoneal fibrosis, we further examined the expression of TGF- $\beta 1 / \mathrm{Smad} 2 / 3$ in a PD mouse model in $W T$ and $J l p^{-/-}$ mice. The expression of TGF- $\beta 1$ and the phosphorylation of Smad2/3 were higher in PD-treated mice compared with PBS-treated mice and further increased in $J l p^{-1-}$ mice (Fig. 3a). Next, we performed ELISA to investigate the secretion of TGF- $\beta 1$. We found that HGPDS induced TGF- $\beta 1$ secretion in HMrSV 5 cells. Knockdown of JLP induced higher level of TGF- $\beta 1$ secretion (Fig. $3 b$ ).

\section{Defective Jlp Expression Resulted in a Distinct EMT}

Phenotype in Peritoneum Mesothelial upon HGPDS

Treatment

EMT is closely linked to peritoneal fibrosis. We found the expression of $\mathrm{N}$-cadherin and vimentin was increased, and the expression of E-cadherin was decreased in HGPDS-treated mice and HMrSV5 cells (Fig. 4a, b). Moreover, the alteration of vimentin and E-cadherin was more pronounced in $J l p^{-/-}$mice and HMrSV5 cells transfected with Jlp siRNA under HGPDS treatment. However, Jlp deficiency resulted in the inhibition of N-cadherin compared with the wild-type group under HGPDS treatment (Fig. 4a, b). Similar results were obtained in Jlp knockdown cells treated with HGPDS (Fig. 4a, b). IF staining showed similar alterations in the expression of EMT markers (Fig. 4c). Downregulation of E-cadherin may result in loss of cell-cell contact inhibition and disconnection of intercellular connections, which work together to induce EMT [24]. We found that the migrating cells in the denuded zone reduced by HGPDS were increased with Jlp siRNA (Fig. 4d). These data demonstrated that Jlp promotes peritoneal fibrosis by aggravating EMT.

\section{Loss of Jlp Led to Excessive Activation of Autophagy} and Apoptosis in Both Peritoneal Fibrosis Mice Model and HGPDS-Treated HMrSV5 Cells

HGPDS treatment overactivated autophagy by increasing the expression of LC3-II and Beclin1 and decreasing the expression of p62/SQSTM1 in the peritoneal membrane and HMrSV 5 cells and was more pronounced in $J l p^{-/-}$mice and Jlp knockdown HMrSV5 cells (Fig. 5a,
Fig. 3. Inhibiting JLP promotes activation of the TGF- $\beta /$ Smad signaling pathway in HGPDS-induced fibrotic responses. a The protein expression of TGF- $\beta 1 / \mathrm{Smad} 2 / 3$ in HGPDS-induced peritoneal fibrosis in WT mice and $J l p^{-1-}$ mice was measured by Western blotting. GAPDH was set as loading control. $n=3$. b TGF- $\beta 1$ produced by HMrSV 5 cells was determined by ELISA. $n$ $=3$. NS, no significant difference. ${ }^{*} p<0.05$, ** $p<0.01, * * * p<0.001$.
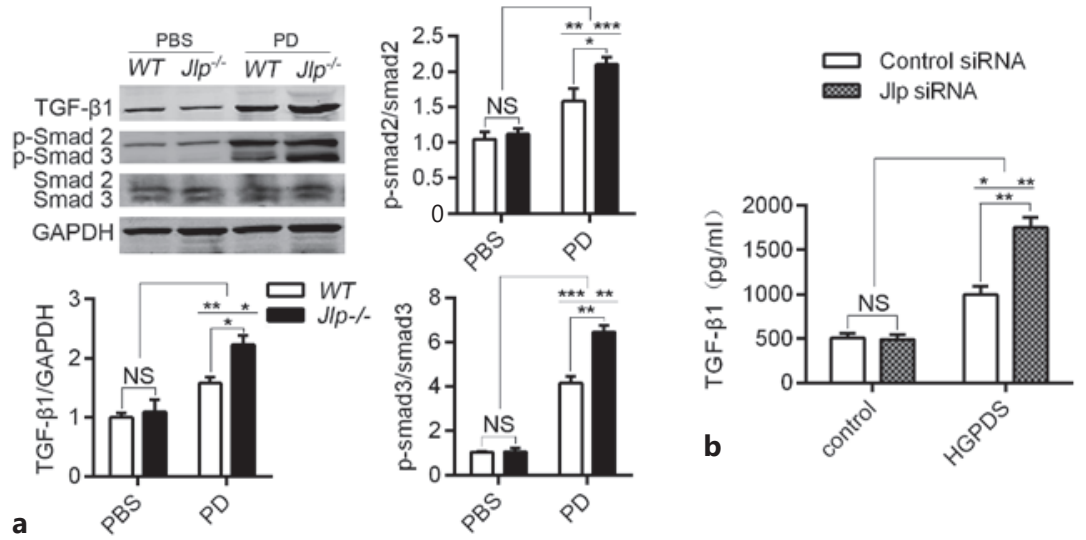

Fig. 2. Jlp downregulation exacerbates HGPDS-induced fibrotic responses in HMrSV5 cells. a Western blotting analyzed the expression of JLP and fibrotic markers, fibronectin, collagen-I, vimentin, and $\alpha$-SMA in the indicated cell samples. GAPDH was set as loading control. $n=3$. b Representative photographs (5 visual

fields for each tissue analyzed) of IF staining for JLP and collagen$\mathrm{I}$ in the indicated cells. Cell nuclei were visualized by co-staining with DAPI. Scale bar, $50 \mu \mathrm{m}$. NS, no significant difference. ${ }^{*} p<$ $0.05,{ }^{* *} p<0.01,{ }^{* * *} p<0.001$. 
Fig. 4. Defective Jlp expression resulted in a distinct EMT phenotype in peritoneum mesothelial upon HGPDS treatment. a, b The protein expression of EMT markers (N-cadherin, E-cadherin, and vimentin) by Western blotting in HGPDS-induced fibrotic responses in vivo and in vitro. GAP$\mathrm{DH}$ was set as loading control. $n=3$. c IF staining ( 5 visual fields for each tissue analyzed) shows the expression of EMT markers in HMrSV 5 cells. d HMrSV 5 cells were plated in 12-well plates and transfected with Jlp siRNA or control siRNA. Then, the motility ability of HMrSV 5 cells was measured by wound-healing assay using an inverted microscope. Scale bar, $50 \mu \mathrm{m}$. NS, no significant difference. ${ }^{*} p<0.05,{ }^{* *} p<$ $0.01,{ }^{* * *} p<0.001$.
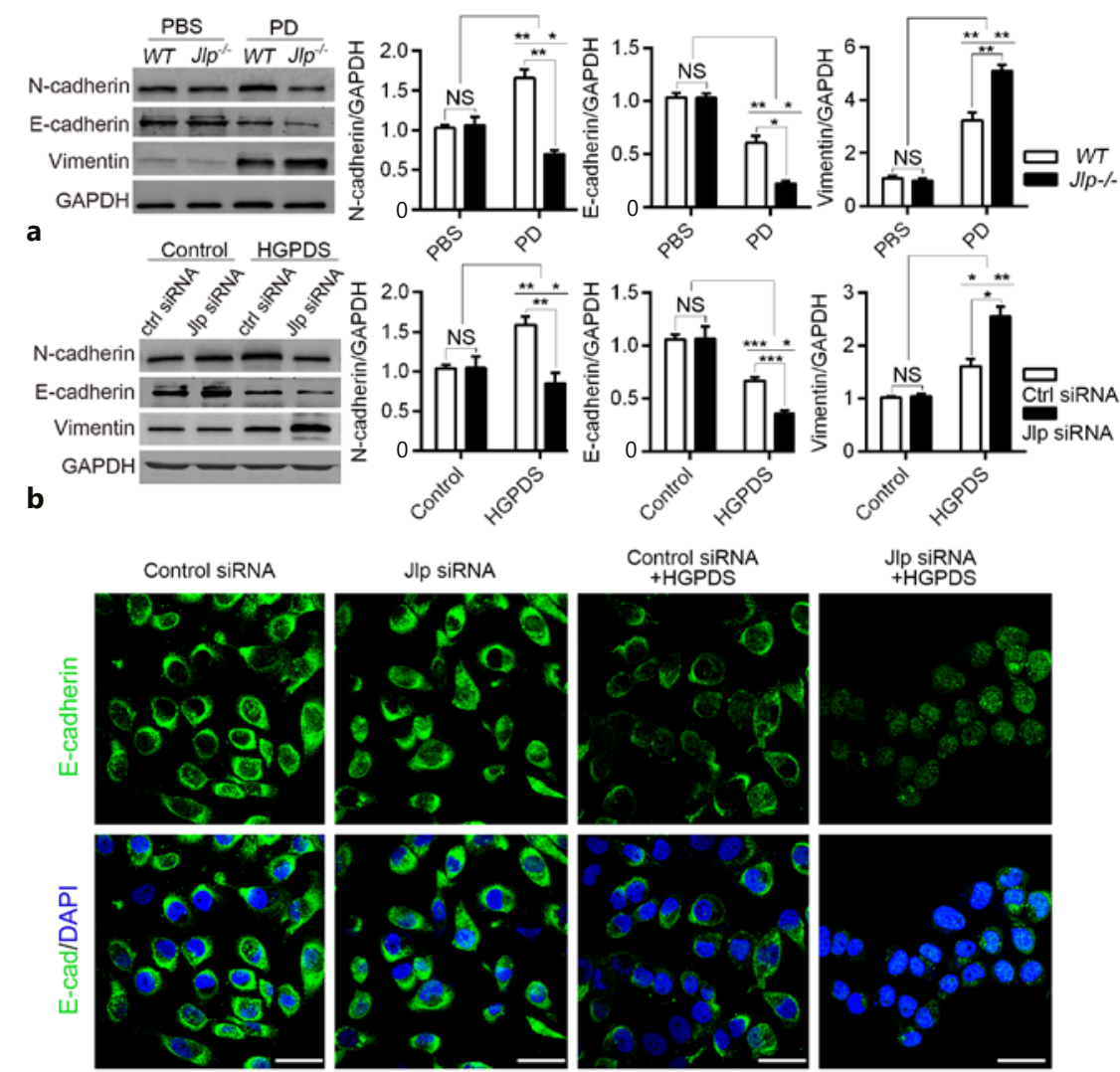

Control siRNA
+ HGPDS

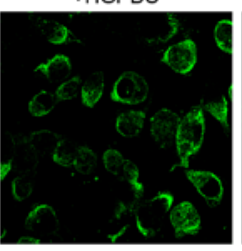

Jlp siRNA
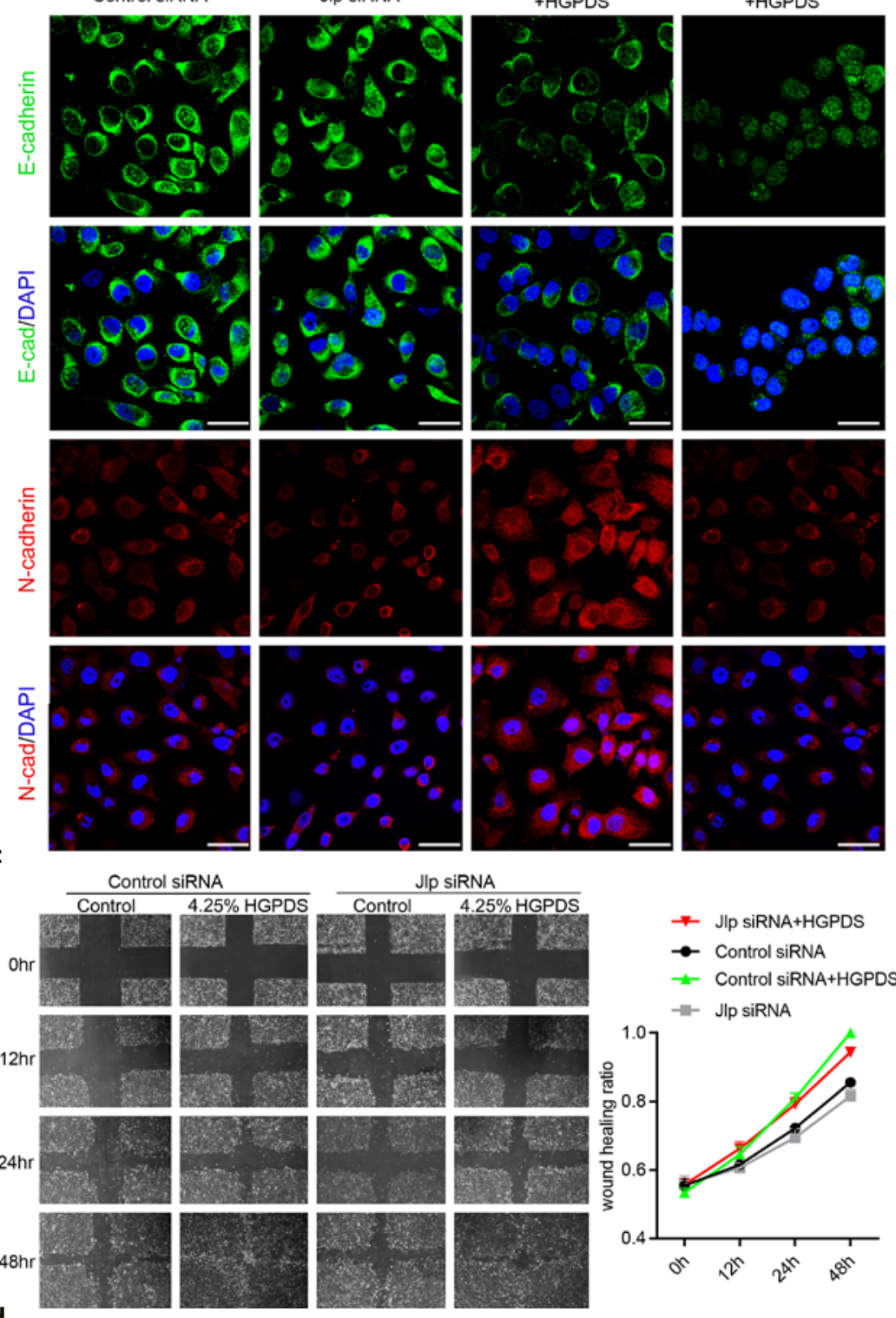

$\rightarrow$ JIp siRNA+HGPDS

$\rightarrow$ Control siRNA

$\leadsto$ Control siRNA+HGPDS

Jlp siRNA

d

Tian/Zhang/Wang/Deng/Peng/Liang/ Ding/Shen/Wang 


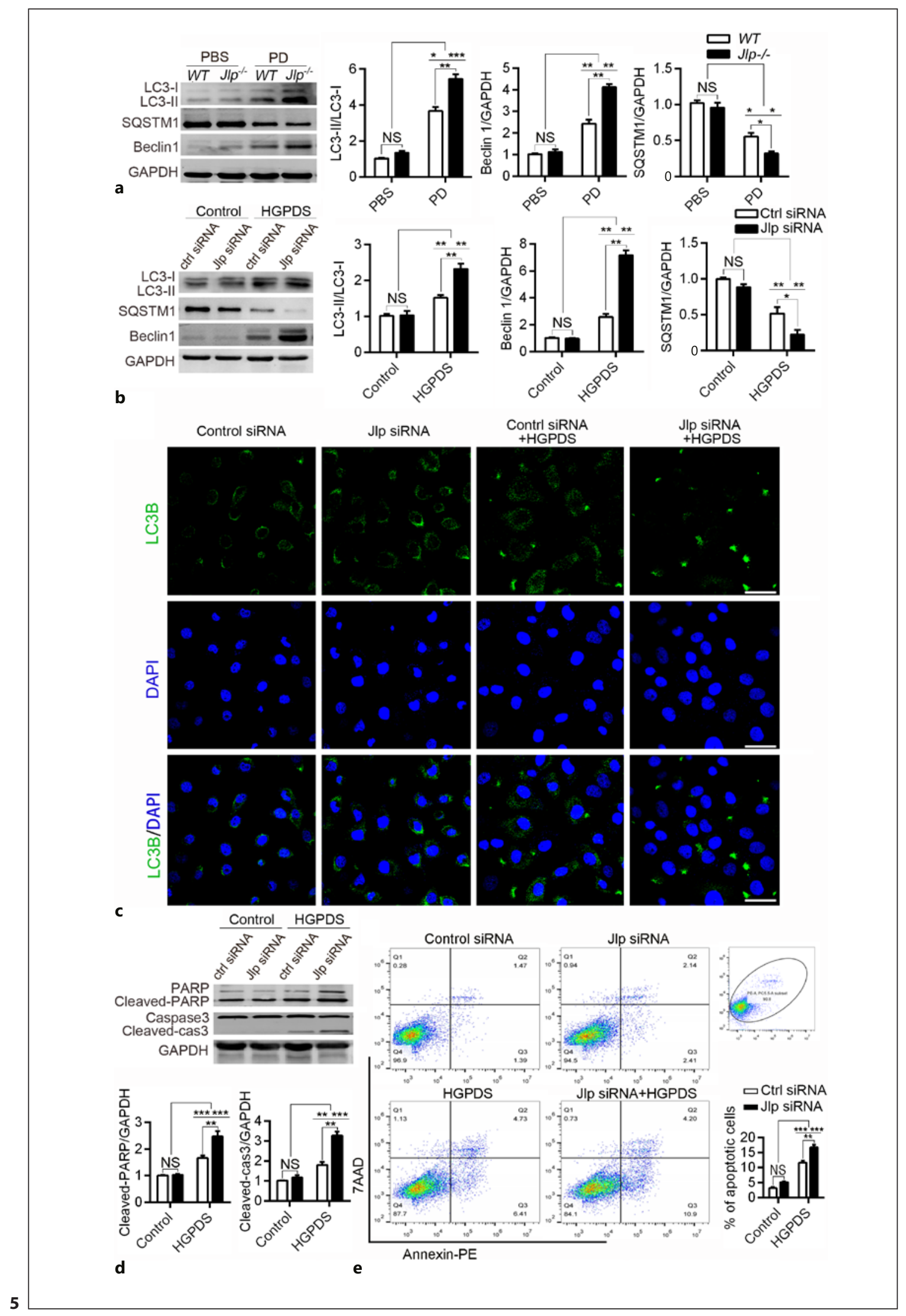

(For legend see next page.) 
b). Additionally, HGPDS-induced autophagy characterized by the LC 3 pellet in HMrSV 5 cells was examined by IF staining (Fig. 5c), and the changes in the LC3 pellet were consistent with the Western blotting results. In addition, we detected the apoptosis of HMrSV5 cells by Western blotting and flow cytometry. Compared with the control group, HGPDS increased the levels of cleaved caspase 3 and caspase downstream effector PARP. And, knockdown of Jlp further increased the cleaved caspase 3 and cleaved PARP under HGPDS treatment (Fig. 5d). Consistent with these findings, cell apoptosis was elevated in the HGPDS-treated HMrSV5 cells compared with control cells and further increased when Jlp was knocked down by siRNA (Fig. 5e).

\section{Discussion}

Peritoneal fibrosis, a severe pathological architecture change of the peritoneum underlying the disastrous clinical outcomes of UF failure and cardiovascular diseases, has been regarded as an irreversible process featured with enlargement of the submesothelial area, excess accumulation of extracellular matrix components (ECMs), increment of blood vessels, and proliferation of myofibroblasts in the peritoneal mesothelium. Although the mechanism of peritoneal fibrosis is not fully understood, the complex interplay between several molecules and cell types, which is mainly triggered by long-term exposure to bioincompatible PD fluids, and in some cases by repeated episodes of peritonitis, has been described in peritoneal fibrosis development. Notably, TGF- $\beta /$ Smad signaling has been widely considered as the key fibrogenic molecular machinery in peritoneal fibrosis, and mesothelial cells act as not only the primary victim but also the active driver during peritoneal fibrosis progression $[25,26]$.

Fig. 5. Loss of Jlp incurred excessive activation of autophagy and apoptosis both in peritoneal fibrosis mice model and HGPDStreated HMrSV5 cells. a, b The expression levels of autophagyrelated proteins (LC3, Beclin 1, and p62/SQSTM1) in the indicated peritoneal membrane and cell samples were analyzed by Western blotting. GAPDH was set as loading control. $n=3$. c IF staining (5 visual fields for each tissue analyzed) shows the expression of the LC3 pellet in HMrSV 5 cells. Scale bar, $50 \mu \mathrm{m}$. d The levels of caspase 3, cleaved caspase 3, PARP, and cleaved PARP protein in HMrSV 5 cells from different groups were analyzed by Western blotting. e HGPDS-induced apoptosis of HMrSV5 cells with Jlp siRNA or control siRNA was detected by flow cytometry. $n=3$. NS, no significant difference. ${ }^{*} p<0.05,{ }^{* *} p<0.01,{ }^{* * *} p<0.001$.
Scaffold proteins are important regulatory factors in many signaling pathways and are intensively involved in organ fibrosis $[27,28]$. JLP, one of the scaffold proteins, contributes to malignancy by promoting cellular proliferation, migration, and colony formation $[14,15]$. A previous study showed that JLP could be involved in promoting tumorigenesis and tumor progression [14]. Our previous study found that JLP was downregulated in renal tissues of renal fibrosis mice models and patients with chronic kidney disease. Considering the potential opposite mechanisms between organ fibrosis and tumors, we further investigated the role of JLP in peritoneal fibrosis. The most important finding of this study is the anti-fibrotic effect of JLP in peritoneal fibrosis. We established an HGPDS-induced peritoneal fibrosis mouse model and found that JLP was downregulated in the fibrotic peritoneal membrane. Moreover, Jlp depletion exacerbated peritoneal fibrosis in vivo and in vitro (Fig. 1, 2). Therefore, it is reasonable to view JLP as a potential anti-fibrosis factor in peritoneal fibrosis.

TGF- $\beta 1$ regulates cell physiology, proliferation, and differentiation and plays a critical role in the pathogenesis and development of various diseases [5]. Previous studies indicated that TGF- $\beta 1$ is a novel profibrotic factor that enhances the expression of profibrotic genes through a novel signaling cascade $[4,5]$. It has been reported that blocking the TGF- $\beta 1$ signaling pathway could significantly attenuate angiogenesis and peritoneal fibrosis in PD mouse models [29]. Thus, inhibiting or blocking the TGF- $\beta 1 /$ Smad signaling pathway has become a key strategy in peritoneal fibrosis treatment. Although TGF- $\beta$ has important immunological and regulatory functions, no TGF- $\beta$ inhibitor has achieved successful anti-fibrotic effects in clinical practice. Our study indicated that JLP can be a potential therapeutic target for peritoneal fibrosis and even various organ fibrosis by blocking the TGF- $\beta 1 /$ Smad signaling pathway (Fig. 1-3).

PD-related peritoneal fibrosis is identical to EMT of peritoneal mesothelial cells, and the loss of peritoneal membrane function results in UF failure. PD solution induces peritoneal irritation, which promotes peritoneal mesothelial cells to translation into a mesenchymal phenotype, including the downregulation of epithelial markers such as E-cadherin and the upregulation of mesenchymal protein expression, such as $\mathrm{N}$-cadherin, vimentin, and $\alpha$-SMA, a process mediated by TGF- $\beta 1$ [4]. EMT usually refers to a switch of $\mathrm{E}$ - to $\mathrm{N}$-cadherin. In this study, we confirmed that the intercellular adhesion molecule E-cadherin was downregulated and that mesenchymal-associated molecules such as $\mathrm{N}$-cadherin, vimentin, 


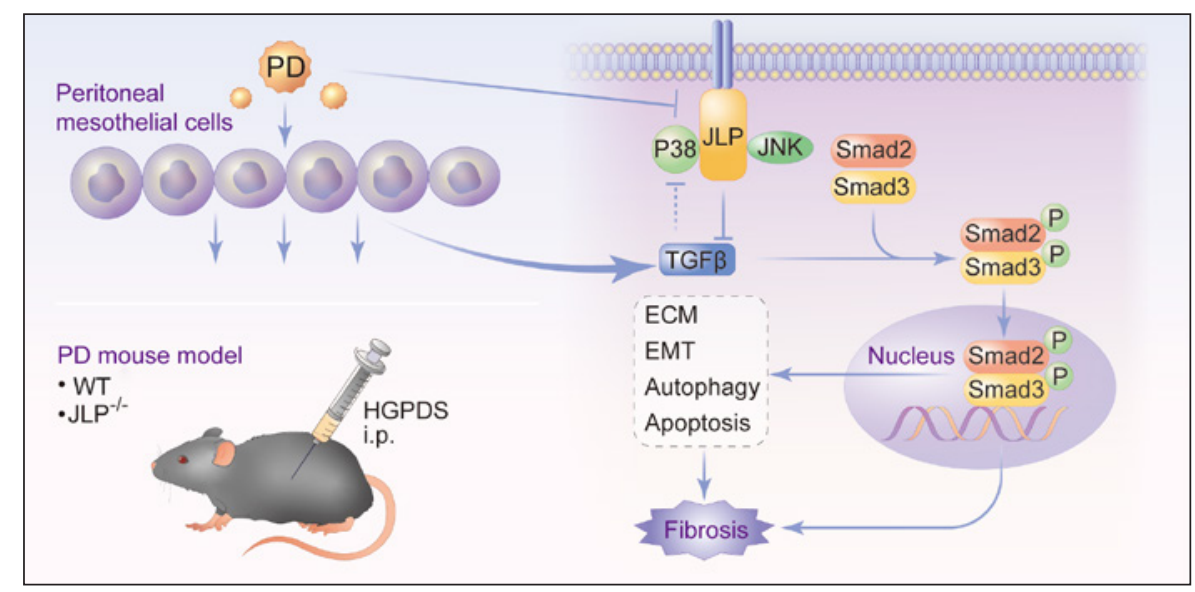

Fig. 6. Schematic illustration of the role of JLP in peritoneal fibrosis. PD treatment results in the release of large amounts of TGF- $\beta$ from peritoneal mesothelial cells. Smad 2 and Smad 3 are activated by phosphorylation and form complexes in response to TGF- $\beta$ stimulation and translocate into the nucleus to regulate the transcription of target genes in collaboration with various co-activators and co-repressors. The TGF- $\beta /$ Smad signaling pathway plays a critical role in peritoneal fibrosis and promotes the deposition of ECM, EMT, autophagy, and apoptosis which involves the regulation of peritoneal fibrosis. JLP deficiency aggravates peritoneal fibrosis induced by HGPDS. JLP plays a protective role in peritoneal fibrosis by negatively regulating the TGF- $\beta /$ Smad signaling pathway, EMT, autophagy, and apoptosis. and $\alpha$-SMA were upregulated in HGPDS-treated HMrSV5 cells. Interestingly, Jlp deficiency promoted a decrease in $\mathrm{E}$-cadherin and an increase in vimentin and $\alpha$-SMA but a loss of N-cadherin upregulation (Fig. 4). We considered that a decrease or deficiency in JLP leads to the loss of the normal phenotype of peritoneal mesothelial cells, further inducing the partial EMT of peritoneal mesothelial cells, causing loss of the ability to repair and replicate, eventually exacerbating peritoneal membrane damage. Furthermore, the precise mechanism of the downregulation of $\mathrm{E}$-cadherin and the loss of $\mathrm{N}$-cadherin in $\mathrm{Jlp}$ deficiency in peritoneal fibrosis are still under further investigation.

Autophagy is a protective response activated by extracellular stress to clear damaged organelles and macromolecules by lysosomes. However, dysfunctional autophagy results in lysosomal dysfunction and impaired clearance of autophagosomes and leads to cell death [30]. Autophagy is implicated in the pathological process of organ fibrosis (including liver fibrosis, renal fibrosis, pulmonary fibrosis, and myocardial fibrosis) [31-34]. Autophagy activation has also been observed in peritoneal fibrosis, but its exact role in fibrosis development remains controversial. Previous studies demonstrated that the induction of autophagy could be a protective mechanism, which resulted in the mitigation of peritoneal fibrosis via curbing mitochondrial reactive oxygen species production and inflammatory damage $[35,36]$. In addition, another group reported that autophagy induction by rapamycin significantly reduced TGF- $\beta 1$-induced
ECM deposition [37]. However, several studies also demonstrated the opposite role of autophagy in promoting organ fibrosis $[38,39]$. In long-term PD treatment, the upregulation of TGF- $\beta 1$, autophagy, and apoptosis perpetually existed and were associated with the progression of peritoneal fibrosis [40]. Our previous studies identified a novel anti-fibrotic molecule of JLP that is exclusively expressed in renal tubular epithelial cells (TECs) in kidney tissue, with the potential to antagonize the TGF- $\beta 1$-elicited effects of ECM production, EMT, autophagy activation, apoptosis, and cycle arrest in TECs in kidney fibrosis progression. Inhibition of autophagy by gaining expression of Jlp in TECs significantly retarded the fibrotic lesions both in vitro and in vivo [18]. Together with the similar findings of the protective role of Jlp in peritoneal fibrosis reported in this study, we concluded that permanent and overactivation of autophagy, a result partly due to JLP loss, might promote the development of peritoneal fibrosis.

In addition, apoptosis is also known as programmed cell death, which is an essential characteristic in the initiation, propagation, and resolution of peritoneal fibrosis. Monolayer peritoneal mesothelial cells play an irreplaceable role in peritoneal filtration. The stimulation of biological incompatibility PD solution, peritonitis, and uremia itself activates caspase-dependent cascades, promoting apoptosis and peritoneal fibrosis. In the fibrotic response, apoptotic cells release chemokines and cytokines, leading to inflammation and fibrosis. Furthermore, 
overactivation of autophagy is involved in enhancing apoptosis [41]. Several key factors, including p53, Beclin1, and Bcl-2 family members, have been revealed to be involved in regulating autophagy and apoptosis [42-44]. For example, Beclin 1 is cleaved by activated caspase, and anti-apoptosis proteins (Bcl-2/Bcl-XL) form a complex with Beclin1 to participate in the regulation of anti-autophagy and anti-apoptosis. In general, the interaction between autophagy and apoptosis is complex. Activation of autophagy can adjust apoptotic cell death, and on the contrary, components of apoptotic pathways can regulate autophagy through key proteins.

In summary, we demonstrated that JLP could play an important role in the pathophysiology of peritoneal membrane dysfunction through multiple mechanisms, including the TGF- $\beta /$ Smad signaling pathway, EMT, autophagy, and apoptosis (Fig. 6). JLP may have a potential therapeutic benefit in preventing and treating patients with peritoneal fibrosis on long-term PD.

\section{Statement of Ethics}

All protocols were approved by the Animal Ethics Review Board of Wuhan University and performed in accordance with the guidelines of the National Health and Medical Research Council of China. IACUC Issue No. WDRM20190701.

\section{Conflict of Interest Statement}

The authors declare no conflicts of interest.

\section{Funding Sources}

This research was funded by the National Natural Science Fund of China (No. 81370800 and 81800614) and the Hubei Province Major Scientific and Technological Special Project (No. 2019ACA137).

\section{Author Contributions}

Maoqing Tian, Lu Zhang, Bo Shen, and Huiming Wang designed the research. Maoqing Tian, Cancan Peng, and Meili Deng performed the experiments. Yujuan Wang and Wei Liang provided clinical consultations and methodology. Maoqing Tian, Lu Zhang, Guohua Ding, and Huiming Wang wrote and edited the manuscript. All authors participated in the discussion of this manuscript and agreed to the published version of the manuscript.

\section{Data Availability Statement}

The data presented in this study are available on request from the corresponding author.

\section{References}

1 Li PK, Chow KM, Van de Luijtgaarden MW, Johnson DW, Jager KJ, Mehrotra R, et al. Changes in the worldwide epidemiology of peritoneal dialysis. Nat Rev Nephrol. 2017 Feb;13(2):90-103.

2 Wang Y, Zhan X, Chen Y, Yang M, Yan C, Qinkai C. Reasons for the dropout of peritoneal dialysis patients. Chin J Nephrol. 2019;(4):275-80.

3 Mehrotra R, Devuyst O, Davies SJ, Johnson DW. The current state of peritoneal dialysis. J Am Soc Nephrol. 2016 Nov;27(11):3238-52.

4 Kim YC, Kim KH, Lee S, Jo JW, Park JY, Park MS, et al. ST2 blockade mitigates peritoneal fibrosis induced by TGF- $\beta$ and high glucose. J Cell Mol Med. 2019 Oct;23(10):6872-84.

5 Budi EH, Duan D, Derynck R. Transforming growth factor-beta receptors and Smads: regulatory complexity and functional versatility. Trends Cell Biol. 2017 Sep;27(9):658-72.

6 Meng X, Nikolic-Paterson DJ, Lan HY. TGF- $\beta$ : the master regulator of fibrosis. Nat Rev Nephrol. 2016 Apr;12(6):325-38.

7 Nieto MA, Huang RY, Jackson RA, Thiery JP. EMT: 2016. Cell. 2016 Jun;166(1):21-45.

8 Liu Y, Dong Z, Liu H, Zhu J, Liu F, Chen G. Transition of mesothelial cell to fibroblast in peritoneal dialysis: EMT, stem cell or bystander? Perit Dial Int. 2015 Jan-Feb;35(1):14-25.

9 Mizushima N. A brief history of autophagy from cell biology to physiology and disease. Nat Cell Biol. 2018 May;20(5):521-7.

10 Dhanasekaran DN, Kashef K, Lee CM, Xu H, Reddy EP. Scaffold proteins of MAP-kinase modules. Oncogene. 2007 May;26(22):3185202.

11 Iwanaga A, Wang G, Gantulga D, Sato T, Baljinnyam T, Shimizu K, et al. Ablation of the scaffold protein JLP causes reduced fertility in male mice. Transgenic Res. 2008 Dec; 17(6):1045-58.

12 Xie C, Fu L, Liu N, Li Q. Overexpression of SPAG9 correlates with poor prognosis and tumor progression in hepatocellular carcinoma. Tumour Biol. 2014 Aug;35(8):7685-91.

13 Yi F, Ni W, Liu W, Pan X, Han X, Yang L, et al. SPAG9 is overexpressed in human astrocytoma and promotes cell proliferation and invasion. Tumor Biol. 2013 Oct;34(5):284955.

$14 \mathrm{HaJH}$, Yan M, Gomathinayagam R, Jayaraman $M$, Husain S, Liu J, et al. Aberrant expression of JNK-associated leucine-zipper protein, JLP, promotes accelerated growth of ovarian cancer. Oncotarget. 2016 Nov;7(45):72845-59.

15 Kanojia D, Garg M, Gupta S, Gupta A, Suri A. Sperm-associated antigen 9 is a novel biomarker for colorectal cancer and is involved in tumor growth and tumorigenicity. Am J Pathol. 2011 Mar;178(3):1009-20.

16 Fu MM, Holzbaur EL. Integrated regulation of motor-driven organelle transport by scaffolding proteins. Trends Cell Biol. 2014 Oct; 24(10):564-74.

17 Qiang F, Huiming W. Role of scaffolding protein JLP on the progression of renal interstitial fibrosis in mice model of unilateral ureteral obstruction and its underlying mechanism. Chin J Nephrol. 2016;32(1):30-6.

18 Yan Q, Zhu K, Zhang L, Fu Q, Chen Z, Liu S, et al. A negative feedback loop between JNKassociated leucine zipper protein and TGFbetal regulates kidney fibrosis. Commun Biol. 2020 Jun;3(1):288.

19 Helmke A, Nordlohne J, Balzer MS, Dong L, Rong S, Hiss M, et al. CX3CL1-CX3CR1 interaction mediates macrophage-mesothelial cross talk and promotes peritoneal fibrosis. Kidney Int. 2019 Jun;95(6):1405-17. 
20 Guo Y, Wang L, Gou R, Wang Y, Shi X, Pang $\mathrm{X}$, et al. SIRT1-modified human umbilical cord mesenchymal stem cells ameliorate experimental peritoneal fibrosis by inhibiting the TGF- $\beta /$ Smad3 pathway. Stem Cell Res Ther. 2020 Aug;11(1):362.

21 Liu J, Jiang C-M, Feng Y, Zhu W, Jin B, Xia $\mathrm{Y}-\mathrm{Y}$, et al. Rapamycin inhibits peritoneal fibrosis by modifying lipid homeostasis in the peritoneum. Am J Transl Res. 2019 Mar; 11(3):1473-85.

22 Pavlyukov MS, Yu H, Bastola S, Minata M, Shender VO, Lee Y, et al. Apoptotic cell-derived extracellular vesicles promote malignancy of glioblastoma via intercellular transfer of splicing factors. Cancer Cell. 2018 Jul;34(1):119-35.

23 Zhang X, Qi Z, Yin H, Yang G. Interaction between p53 and Ras signaling controls cisplatin resistance via HDAC4- and HIF-1amediated regulation of apoptosis and autophagy. Theranostics. 2019 Jan;9(4):1096-114.

24 Ghosh A, Degyatoreva N, Kukielski C, Story S, Bhaduri S, Maiti K, et al. Targeting miRNA by tunable small molecule binders: peptidic aminosugar mediated interference in miR-21 biogenesis reverts epithelial to mesenchymal transition. Medchemcomm. 2018 Jun;9(7):1147-54.

25 Davies SJ. Unraveling the mechanisms of progressive peritoneal membrane fibrosis. Kidney Int. 2016 Jun;89(6):1185-7.

26 Zhou Q, Bajo MA, Del PG, Yu X, Selgas R. Preventing peritoneal membrane fibrosis in peritoneal dialysis patients. Kidney Int. 2016 Sep;90(3):515-24.

27 Kim-Kaneyama JR, Lei XF, Arita S, Miyauchi A, Miyazaki T, Miyazaki A. Hydrogen Peroxide-Inducible Clone 5 (Hic-5) as a potential therapeutic target for vascular and other disorders. J Atheroscler Thromb. 2012;7(19) $601-7$.
28 Diviani D, Osman H, Reggi E. A-kinase anchoring protein-Lbc: a molecular scaffold involved in cardiac protection. J Cardiovasc Dev Dis. 2018 Feb;5(1):12.

29 Loureiro J, Aguilera A, Selgas R, Sandoval P, Albar-Vizcaino P, Perez-Lozano ML, et al. Blocking TGF-beta 1 protects the peritoneal membrane from dialysate-induced damage. J Am Soc Nephrol. 2011 Sep;22(9): 1682-95.

30 Hazari Y, Bravo-San Pedro JM, Hetz C, Galluzzi L, Kroemer G. Autophagy in hepatic adaptation to stress. J Hepatol. 2020 Jan;72(1): $183-96$.

31 Zhao XC, Livingston MJ, Liang XL, Dong Z. Cell apoptosis and autophagy in renal fibrosis. Adv Exp Med Biol. 2019;1165:557-84.

32 Tseng YJ, Dong L, Liu YF, Xu N, Ma W, Weng $\mathrm{SQ}$, et al. Role of autophagy in chronic liver inflammation and fibrosis. Curr Protein Pept Sci. 2019;20(8):817-22.

$33 \mathrm{Lv}$ X, Li K, Hu Z. Autophagy and pulmonary fibrosis. Adv Exp Med Biol. 2020;1207:56979.

34 Lu C, Yang Y, Zhu Y, Lv S, Zhang J. An intervention target for myocardial fibrosis: autophagy. Biomed Res Int. 2018 Apr;2018: 6215916.

35 Wu J, Li X, Zhu G, Zhang Y, He M, Zhang J. The role of resveratrol-induced mitophagy/ autophagy in peritoneal mesothelial cells inflammatory injury via NLRP3 inflammasome activation triggered by mitochondrial ROS. Exp Cell Res. 2016 Feb 1;341(1):42-53.

36 Yang L, Fan Y, Zhang X, Liu J, Ma J. Effect of $1,25(\mathrm{OH}) 2 \mathrm{D} 3$ on high glucoseinduced autophagy inhibition in peritoneum. Mol Med Rep. 2017 Nov;16(5):7080-5.
37 Li S, Peng F, Gong W, Wu J, Wang Y, Xu Z, et al. Dimethylaminomicheliolide ameliorates peritoneal fibrosis through the activation of autophagy. J Mol Med. 2019 May; 97(5):659-74.

38 Li J, Zhao TT, Zhang P, Xu CJ, Rong ZX, Yan $\mathrm{ZY}$, et al. Autophagy mediates oral submucous fibrosis. Exp Ther Med. 2016 May;11(5): 1859-64.

39 Shu S, Zhu J, Liu Z, Tang C, Cai J, Dong Z. Endoplasmic reticulum stress is activated in post-ischemic kidneys to promote chronic kidney disease. EBioMedicine. 2018 Nov;37: 269-80.

$40 \mathrm{Wu}$ J, Xing C, Zhang L, Mao H, Chen X, Liang $\mathrm{M}$, et al. Autophagy promotes fibrosis and apoptosis in the peritoneum during long-term peritoneal dialysis. J Cell Mol Med. 2018 Feb; 22(2):1190-201.

41 Zhang M, Zhao G, Hou Y, Zhong S, Xu L, Li $\mathrm{F}$, et al. Racl conditional deletion attenuates retinal ganglion cell apoptosis by accelerating autophagic flux in a mouse model of chronic ocular hypertension. Cell Death Dis. 2020 Sep 10;11(9):734.

42 Qi R, Wang J, Jiang Y, Qiu Y, Xu M, Rong $\mathrm{R}$, et al. Snail-induced partial epithelial: mesenchymal transition orchestrates p53p21-mediated G2/M arrest in the progression of renal fibrosis via NF- $\kappa \mathrm{B}-$ mediated inflammation. Cell Death Dis. 2021 Jan 5; 12(1):44.

43 Ham SJ, Lee D, Yoo H, Jun K, Shin H, Chung J. Decision between mitophagy and apoptosis by Parkin via VDAC1 ubiquitination. Proc Natl Acad Sci U S A. 2020 Feb;117(8):428191.

44 Qiao H, Zhang L, Fang D, Zhu Z, He W, Hu $\mathrm{L}$, et al. Surmounting tumor resistance to metallodrugs by co-loading a metal complex and siRNA in nanoparticles. Chem Sci. 2021 Feb;12(12):4547-56. 